\title{
Investigation of carbon nanotube antennas using thin wire integral equations
}

\author{
N. Fichtner, X. Zhou, and P. Russer \\ Institute for High Frequency Engineering, Technische Universität München, Arcisstr. 21, 80333 München, Germany
}

\begin{abstract}
In this paper the characteristics of small carbon nanotube (CNT) dipole antennas are investigated on the basis of the thin wire Hallén integral equation (IE). A surface impedance model for the CNT is adopted to account for the specific material properties resulting in a modified kernel function for the integral equation. A numerical solution for the IE gives the current distribution along the CNT. From the current distribution the antenna driving point impedance and the antenna efficiency are computed. The presented numerical examples demonstrate the strong dependence of the antenna characteristics on the used material and show the limitations of nanoscale antennas.
\end{abstract}

\section{Introduction}

The electromagnetic behavior of nanoscale circuits, transmission lines and antenna elements is of special interest for future highly integrated electrical circuits operating at extremely high clock rates. Especially the wireless transmission of signals between nanoscale circuit components and the microelectronic periphery is a key issue in present-day nanoelectronics. Carbon nanotubes (CNT) are interesting components for further miniaturization of electrical circuits and can be used as signal transmission lines as well as for antenna elements. Their small dimensions however demand a special treatment. Usually in antenna theory the metallic conductors in the investigated structures are treated as perfect electric conductors (Balanis, 1997). This assumption does not hold at nanoscale dimensions. Here the inherent material losses are considerable and impose limitations to the used materials like copper or aluminum for components at the nanoscale. Carbon nanotubes (CNT) exhibit ballistic transport over length of several $\mu \mathrm{m}$ and have a relaxation time which is approximately 50 times greater than in copper (Jishi et al., 1993).

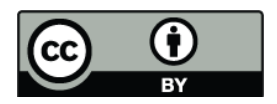

Correspondence to: N. Fichtner (fichtner@tum.de)
This offers the applicability of CNTs for the transmission and reception of electromagnetic signals.

\section{Theory}

\subsection{Integral equation method}

An incident electromagnetic wave induces an electric current $I_{z}\left(z^{\prime}\right)$ flowing in the thin wire dipole element (transverse currents are neglected because of the small diameter). From the current distribution and the excitation the antenna driving point impedance and the antenna efficiency can be computed. The two classical integral equations for linear antennas are the Pocklington's and Hallén's integral equations (Balanis, 1997). As Hallén's IE exhibit better stability properties a numerical solution thereof is less error-prone and will be used in the following (Sarkar, 1983). Hallén's integral equation is given by

$\int_{-l / 2}^{+l / 2} K\left(z, z^{\prime}\right) I_{z}\left(z^{\prime}\right) d z^{\prime}=\frac{-j}{Z_{F 0}}\left[c_{1} \cos (k z)+c_{2} \sin (k|z|)\right]$

with $k$ as the wavenumber. The integral kernel $K\left(z, z^{\prime}\right)$ will be discussed later in Sect. 2.2. For a delta gap excitation in the dipole center the constant $c_{2}$ is given by $c_{2}=V_{0} / 2$ with $V_{0}$ as the driving voltage and $c_{1}$ is obtained from the condition $I_{z}( \pm l / 2)=0$. For a numerical solution of Eq. (1) a Method of Moments (MoM) approach is applied (Balanis, 1997). There the unknown current density $I_{z}\left(z^{\prime}\right)$ is replaced by a series of known expansion functions. As expansion functions pulsefunctions

$u_{i}(z)= \begin{cases}1 & \text { for } z_{i}-\frac{\Delta z}{2}<z<z_{i}+\frac{\Delta z}{2} \\ 0 & \text { elsewhere }\end{cases}$

are used with $\Delta z=l / N$. The coefficients of the expansion of $I_{z}\left(z^{\prime}\right)$ are chosen such that at specific sampling points

Published by Copernicus Publications on behalf of the URSI Landesausschuss in der Bundesrepublik Deutschland e.V. 

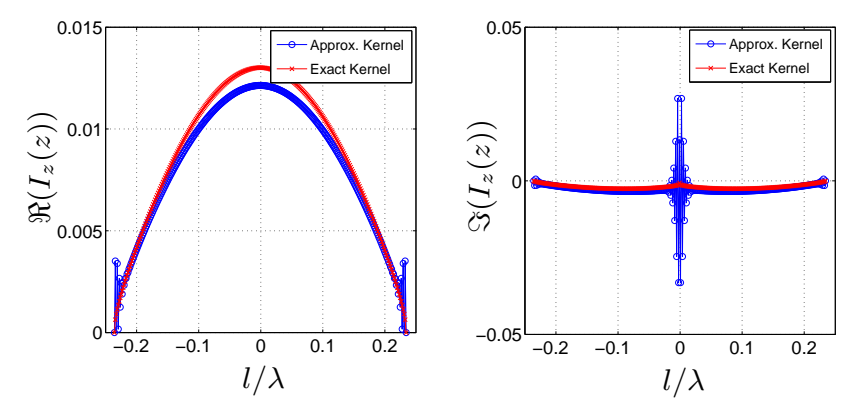

Fig. 1. Current distribution along the dipole for approx. kernel (blue line) and exact kernel (red line).

$z_{i}=(2 i-1) \Delta z / 2$ the IE in Eq. (1) is fulfilled (point matching). This results in a linear system of equations which can be solved by matrix inversion.

\subsection{Modified kernel function}

In CNTs the electric current is confined to a thin cylindrical shell and can be computed from the surface conductance $\sigma$ (Slepyan et al., 1999). Hence the total current flowing in the CNT dipole with radius $a$ is given by

$$
\frac{I_{z}(z)}{2 \pi a}=\sigma\left(E_{z}^{s}(z)+E_{z}^{i}(z)\right)
$$

where $E_{z}^{s}$ is the scattered electric field and $E_{z}^{i}$ is the incident electric field. Combination of Eq. (3) with the Hallén integral equation yields

$$
\begin{array}{r}
\left(k^{2}+\partial_{z}^{2}\right) \int_{-l / 2}^{+l / 2} K\left(z, z^{\prime}\right) I_{z}\left(z^{\prime}\right) d z^{\prime} \\
=j \omega \epsilon\left(Z_{i}^{\prime} I_{z}(z)-E_{z}^{i}(z)\right)
\end{array}
$$

where $Z_{i}^{\prime}$ is the impedance per unit length given by

$$
Z_{i}^{\prime}=\frac{1}{2 \pi a \sigma}
$$

In order to solve Eq. (4) in the same way then Eq. (1) the unknown current $I_{z}(z)$ on the right side of Eq. (4) must be eliminated. With the help of the Green's function for the differential operator $\left(k^{2}+\partial_{z}^{2}\right)$ the current term $I_{z}(z)$ can be written as an integral over the dipole length. Thus the new IE comprising the surface conductance is given by

$$
\begin{aligned}
& \int_{-l / 2}^{+l / 2} K^{\prime}\left(z, z^{\prime}\right) I_{z}\left(z^{\prime}\right) d z^{\prime} \\
& =c_{1} \cos (k z)+c_{2} \sin (k|z|)+\frac{\omega \varepsilon}{2 k} e^{-j k|z|} V_{0}
\end{aligned}
$$

with the modified integral kernel

$$
K^{\prime}\left(z, z^{\prime}\right)=K\left(z, z^{\prime}\right)+\frac{e^{-j k\left|z-z^{\prime}\right|}}{2 Z_{F 0} Z_{i}^{\prime}}
$$

Unlike in Eq. (4) in Eq. (6) the current $I_{z}\left(z^{\prime}\right)$ is solely given under the integral with the modified integral kernel $K^{\prime}\left(z, z^{\prime}\right)$ and can be solved in the same way than Eq. (1). Now both constants $c_{1}$ and $c_{2}$ must be chosen such that $I_{z}( \pm l / 2)=0$ is fulfilled.

\subsection{Kernel selection and stability}

In computations with a large discretization number $N$, i.e. a small discretization length $\Delta z$, oscillations in the current distribution are observed near the dipole center and the dipole ends. Fikioris and $\mathrm{Wu}(2001)$ have shown that the current oscillations arise from the approximate kernel function $K_{\text {app }}(z)$ if the number of basis functions $N$ satisfies $N \gg l / a$. The exact and approximate kernels are

$$
K_{\mathrm{ex}}\left(z, z^{\prime}\right)=\frac{1}{2 \pi} \int_{-\pi}^{\pi} \frac{e^{-j k \sqrt{\left(z-z^{\prime}\right)^{2}+4 a^{2} \sin ^{2} \theta / 2}}}{\sqrt{\left(z-z^{\prime}\right)^{2}+4 a^{2} \sin ^{2} \theta / 2}} d \theta
$$

and

$K_{\text {app }}\left(z, z^{\prime}\right)=\frac{e^{-j k \sqrt{\left(z-z^{\prime}\right)^{2}+a^{2}}}}{\sqrt{z^{2}+a^{2}}}$

In Fig. 1 the current distribution along a $0.47 \lambda$ long dipole with radius $a / \lambda=0.007$ and $N=1.5 l / a$ is shown for the approximate and exact kernel. The driving point impedance for the particular cases are $Z_{\text {in,app }}=37.3-j 41 \quad \Omega$ and $Z_{\text {in, ex }}=76.6-j 42 \Omega$. Thus the choice of the Kernel and the number of expansion functions $N$ strongly affects the input impedance. Here the exact kernel is used at the cost of a additional integration which increases the computational effort.

\section{Complex surface conductance}

The complex surface conductance for a CNT was derived by Slepyan et al. (1999). Slepyan solved the single electron Boltzmann transport equation under consideration of the band structure and the impulse quantization in transverse direction. For small radii the CNT conductance can be approximated by

$\sigma_{c n t} \cong-j \frac{2 e_{0}^{2} v_{F}}{\pi^{2} \hbar(\omega-j / \tau)}$

where $e_{0}$ is the elementary charge and $v_{F} \approx 9.71 \cdot 10^{5} \mathrm{~m} / \mathrm{s}$ is the Fermi velocity of graphene. The relaxation time $\tau$ at room temperature is about $\tau=1.4 \cdot 10^{-12} \mathrm{~s}$. In order to compare the characteristics of a CNT dipole with a copper dipole the surface conductance for copper is needed. From Hanson (2005) we get

$$
\sigma_{c u}=-j \frac{e_{0}^{2} N_{e}^{2 D}}{m_{e}\left(\omega-j / \tau_{c u}\right)}
$$

with $N_{e}^{2 D}$ is the number of electrons per $\mathrm{m}^{2}$. 
Table 1. Input impedance and efficiency $\eta$ for perfect conducting (pc), CNT and copper dipole with length $l=0.47 \lambda$. The operating frequency is $100 \mathrm{GHz}$ and $N=51$ for the half dipole $\left(N_{e}^{2 D} \approx 1.93 \cdot 10^{19} 1 / \mathrm{m}^{2}\right)$.

\begin{tabular}{ccc}
\hline Radius $a$ & Input impedance/ $\Omega$ & Efficiency $\eta / \%$ \\
\hline \multirow{3}{*}{$3 \mu \mathrm{m}$} & $Z_{p c}=67-j 19$ & 100 \\
& $Z_{c n t}=140-j 2575$ & $1.6 \cdot 10^{-3}$ \\
& $Z_{c u}=75-j 1727$ & $2.9 \cdot 10^{-2}$ \\
\hline \multirow{3}{*}{$30 \mathrm{~nm}$} & $Z_{p c}=63-j 82$ & 100 \\
& $Z_{c n t}=950-j 7934$ & $1.1 \cdot 10^{-3}$ \\
& $Z_{c u}=666-j 4733$ & $2.6 \cdot 10^{-3}$ \\
$3 \mathrm{~nm}$ & $Z_{p c}=62-j 109$ & 100 \\
& $Z_{c n t}=1819-j 10730$ & $6.8 \cdot 10^{-4}$ \\
& $Z_{c u}=1978-j 7913$ & $7.6 \cdot 10^{-4}$ \\
\hline
\end{tabular}

\section{Results}

Firstly the input impedances and the achievable efficiencies are computed for dipoles at nanometer radii with a length of $l=0.47 \lambda$. Thus the surface conductances defined in Eqs. (10) and (11) are combined with the IE (6) and solved for the unknown current distribution $I_{z}(z)$. The input impedance is obtained from $Z_{i n}=V_{0} / I_{z}(0)$ and the radiation efficiency is given by

$\eta=\frac{R_{i n}}{R_{i n}+R_{h f}} \quad$ with $\quad R_{h f}=\Re\left\{\frac{l}{2 \pi a} \sqrt{\frac{\omega \mu_{0}}{2 \sigma}}\right\}$

and $R_{i n}=\Re\left\{Z_{i n}\right\}$. The results are summarized in Table 1. From Table 1 follows that for smaller radii the impedance values as well as the efficiency of CNT and copper dipoles are comparable. In the present discussion the efficiency of copper dipoles may even decrease at nanometer radii if we account for the additional surface and grain scattering (Hanson, 2005) which is not included in Eq. (11). The conductivity of CNTs however is not affected by surface or grain scattering because of their regular structure and can even be increased up to a factor of 30 if the CNT is doped (Dekker, 1999).

Besides the efficiency the frequency dependence of the input impedance of a CNT dipole is investigated. In Fig. 2 the dipole driving impedance from 0 to $1000 \mathrm{GHz}$ is shown. Remarkable are the positions of the resonances. Usually the first resonance would be at $f=c_{0} / 2 l=7.5 \mathrm{THz}$ whereas here the first resonance is at $f=160 \mathrm{GHz}$. Consequently the wavelength $\lambda$ on the CNT dipole must be reduced by a factor of $\lambda_{0} / \lambda \approx 50$ which is denoted as the slow wave factor (Hanson, $2005)$. This behavior can be explained by the presence of retarded plasmon modes in CNTs (Fichtner and Russer, 2006).

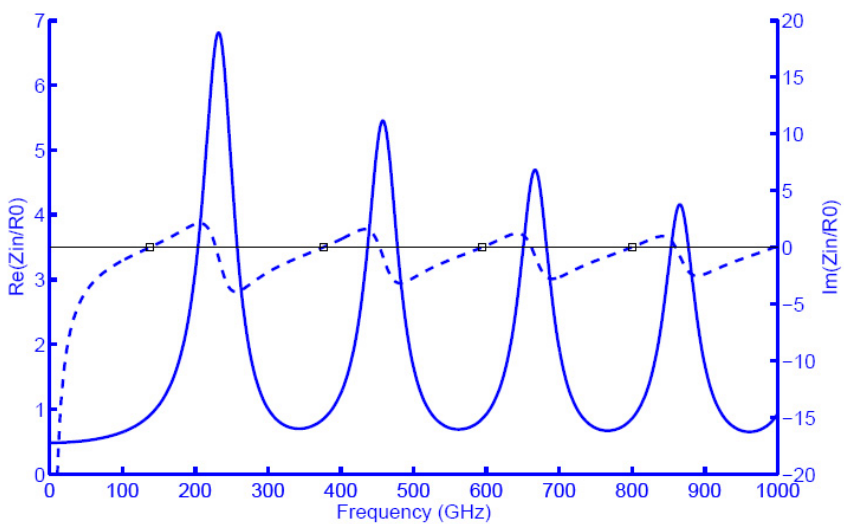

Fig. 2. Normalized input impedance for a CNT nanoantenna for $a=5 \mathrm{~nm}$ and $l=20 \mu \mathrm{m}\left(R_{0}=12.9 \mathrm{k} \Omega\right)$.

\section{Conclusions}

Nanoantennas either of copper or of carbon exhibit inherently a very high resistance per unit length. A lower resistance may be achievable due to doping of the CNTs. The low efficiency of nanoantennas restrict the signal transmission to very short distances as present in e.g. chip-to-chip or on-chip communication systems (Yordanov et al., 2008).

\section{References}

Balanis, C.: Antenna Theory - Analysis and Design, John Wiley \& Sons, 2 edn., 1997.

Dekker, C.: Carbon Nanotubes as molecular quantum wires, Physics Today, 52, 22-28, 1999.

Fichtner, N. and Russer, P.: On the Possibility of Nanowire Antennas, in: Microwave Conference, 36th European, 870-873, 2006.

Fikioris, G. and Wu, T.: On the Application of Numerical Methods to Hallén's Equation, IEEE Trans. Antennas Propagat., 49, 383 392, 2001.

Hanson, G.: Fundamental Transmitting Properties of Carbon Nanotube Antennas, IEEE Trans. Antennas Propagat., 53, 34263435, 2005.

Jishi, R., Dresselhaus, M., and Dresselhaus, G.: Electron-phonon coupling and the electrical conductivity of fullerene nanotubules, Phys. Rev. B, 48, 11 385-11 389, 1993.

Sarkar, T.: A study of the various methods for computing electromagnetic field utilizing thin wire integral equations, Radio Sci., 18, 29-38, 1983.

Slepyan, G., Maksimenko, S., Lakhtakia, A., Yevtushenko, O., and Gusakov, A.: Electrodynamics of carbon nanotubes: Dynamic conductivity, impedance boundary conditions, and surface wave propagation, Phys. Rev. B, 60, 17 136-17 149, 1999.

Yordanov, H., Ivrlac, M. T., Mezghani, A., Nossek, J., and Russer, P.: Computation of the Impulse Response and Coding Gain of a Digital Interconnection Bus, in: Proceedings of the 23rd Annual Review of Progress in Applied Computational Electromagnetics ACES, Niagara Falls, Canada, accepted, 2008. 